\title{
Travel Time Reliability for Urban Networks: Modelling and Empirics
}

\author{
Fangfang Zheng, ${ }^{1,2}$ Xiaobo Liu, ${ }^{1,2}$ Henk van Zuylen, ${ }^{1,2,3,4} \mathrm{Jie} \mathrm{Li}^{4}$ and Chao $\mathrm{Lu}^{1,2}$ \\ ${ }^{1}$ School of Transportation and Logistics, Southwest Jiaotong University, No. 111 Erhuanlu Beiyiduan, Chengdu 610031, China \\ ${ }^{2}$ The National United Engineering Laboratory of Integrated and Intelligent Transportation, Southwest Jiaotong University, \\ No. 111 Erhuanlu Beiyiduan, Chengdu 610031, China \\ ${ }^{3}$ Transport and Planning Department, Delft University of Technology, P.O. Box 5048, 2600 GA Delft, Netherlands \\ ${ }^{4}$ Civil Engineering College, Hunan University, Lushan South Road, Changsha, Hunan Province 410082, China
}

Correspondence should be addressed to Xiaobo Liu; xiaobo.liu@swjtu.cn

Received 9 March 2017; Revised 17 May 2017; Accepted 4 June 2017; Published 9 July 2017

Academic Editor: Yuchuan Du

Copyright (c) 2017 Fangfang Zheng et al. This is an open access article distributed under the Creative Commons Attribution License, which permits unrestricted use, distribution, and reproduction in any medium, provided the original work is properly cited.

The importance of travel time reliability in traffic management, control, and network design has received a lot of attention in the past decade. In this paper, a network travel time distribution model based on the Johnson curve system is proposed. The model is applied to field travel time data collected by Automated Number Plate Recognition (ANPR) cameras. We further investigate the network-level travel time reliability by connecting the network reliability measures such as the weighted standard deviation of travel time rate and the weighted skewness of travel time rate distributions with network traffic characteristics (e.g., the network density). The weighting is done with respect to the number of signalized intersections on a trip. A clear linear relation between the weighted average travel time rate and the weighted standard deviation of travel time rate can be observed for different time periods with time-varying demand. Furthermore, both the weighted average travel time rate and the weighted standard deviation of travel time rate increase monotonically with network density. The empirical findings of the relation between network travel time reliability and network traffic characteristics can be possibly applied to assess traffic management and control measures to improve network travel time reliability.

\section{Introduction}

Urban travel times are well recognized to be variable due to time-varying demand, stochastic capacity, different control measures (e.g., signal control at intersections), and external factors (e.g., weather conditions). The most widely used travel time uncertainty modelling philosophy is based on the concept of probability. With the probability distributions, the reliability analysis of link/route/network travel times can be conducted. In the literature, travel time distribution estimation methods in the urban context can be categorized into three groups: analytical approach, statistical (or numerical) approximation, and simulation.

For the analytical approach, Zheng et al. [1,2] proposed an analytical link and trip travel time distribution model for urban signalized arterials. The model explicitly accounts for the influence of stochastic traffic processes, variation of traffic demand, queue dynamics, and different traffic signal control schemes (including cycle time, green time, and offset) on travel time variability. The "spillback" phenomenon (queued traffic from one intersection affecting operations at adjacent intersections) is also taken into account. The advantage of the analytical model lies in its transferability and applicability in different traffic conditions. However, the main drawback is the complexity of the modelling process for network-wide travel time distribution estimation.

For the statistical (or numerical) methods, normal, lognormal, truncated normal/lognormal, Gamma, and Weibull distributions [3-5] are widely applied to describe travel time reliability (variability). Due to the complexity of urban traffic conditions, a single distribution model could not well represent the travel time distribution of an urban road. A mixture of statistical distribution models $[6,7]$ has been proposed to capture multistate traffic conditions and the influence of 
signal control at intersections for urban roads. Clark and Watling [8] employed the method of moments to calculate various travel time reliability measures (expected travel time, variance, skewness, and kurtosis). The values of these four measures were then fitted to a family of probability densities called "Johnson" curves. In order to capture both the vehicleto-vehicle and day-to-day variability in travel time data, Kim and Mahmassani [9] proposed a Gamma-Gamma mixture distribution model. Based on their proposed model, the heterogeneity within these two variability types under different weather conditions can be described as well.

For the simulation method, Kim et al. [10] employed traffic simulation models to derive travel time distributions under different scenarios considering various demand and supply uncertainty factors, such as weather, traffic incidents, work zones, and traffic control. Chen and Zhou [11] proposed a simulation approach to derive network travel times by solving a traffic equilibrium problem considering demand uncertainty. The network travel time samples were further used to estimate the network travel time distribution and its statistics. The main advantage of the simulation approach lies in its flexibility. However, intensive computation is required to conduct the simulation.

Although the concept of travel time reliability is relatively new in the field of transportation compared to some other engineering fields, various travel time reliability measures have been proposed by researchers, for example, standard deviation, statistical range [12], buffer index [13], 90th or 95th percentile [14], skewness-width [15], travel time budget or total travel time budget [16], and mean-excess total travel time [17]. The choice of the most suitable reliability model depends on the context and purpose for which reliability is evaluated and on the characteristic of the travel time distribution [15]. Skewness is sometimes a relevant characteristic because the more extreme long travel times may deter travelers to choose a certain route and the economic value of extreme long travel times is important [18]. In cases for which skewness in the travel time probability distribution is not an issue, the variance of the travel time is a logical reliability measure. Total travel time budget (TTTB) and mean-excess total travel time (METTT) are two risk measures proposed for assessing transportation network performance under uncertainty $[11,17]$. TTTB is defined as the minimum travel time threshold that satisfies a certain reliability requirement given by decision-makers at certain confidence level. Xu et al. [17] define the METT as the conditional expectation of travel times exceeding the corresponding TTTB at a given confidence level. The numerical example shows that applying METTT in the network design problem under uncertainty lowers the construction cost and reduces the unacceptable risk in the distribution tail substantially while incurring a minor TTTB increase.

Up to now, most research focuses on modelling or measuring link/segment level or route level travel time reliability. Research on network wide travel time reliability is rather limited and mainly based on simulation. Mahmassani et al. [19] used both simulated data and field GPS trajectory data to model travel time reliability. Their investigation with field data is rather limited. Moreover, GPS data is only a sample of the total traffic on the network and no flow data is available in their study area. The question is to what extent the derived relationship between the travel time variability and the network flow or density deduced from GPS sample data can represent the real situation.

The network travel time reliability has been considered as an important aspect in network management, control, and design $[20]$. In $[1,2]$, the authors have developed an analytical travel time distribution model for link and route levels. When it comes to the network level, the previously developed "pure analytical model" is unable to describe the travel time variability. Therefore, in this paper, a network travel time distribution model based on flexible probability densities known as Johnson curves is introduced in Section 2. Different from previous research on travel time reliability modelling with Johnson curves estimated based on the method of moments, we apply a percentile-point method to estimate Johnson curves. The capabilities of Johnson curves are demonstrated with field travel time data. In Section 3, we discuss the calculation of travel time reliability measures for urban networks and their connection with network traffic characteristics (e.g., network traffic density and flow). An illustration of travel time reliability based on empirical data for a real urban network of a city in China using ANPR data is provided as well. Finally, some discussion and conclusions are provided in the last section.

\section{Network Travel Time Distribution}

2.1. Vehicle-to-Vehicle Travel Time Variability from Empirical Observations. Travel times that vehicles experience in the urban network can be influenced by many factors, such as fluctuations in traffic demand and supply, signal control at intersections, turning vehicles from cross streets, bus maneuvers at bus stops, parking vehicles along the roadside, crossing pedestrians and cyclists, and, of course, intermediate stops of the vehicles themselves, for example, for certain activities of the driver along the trip. As a result, urban travel times are rather variable given known traffic condition (traffic flow). Figure 1(a) illustrates the empirical travel time-flow relationship derived from local $5 \mathrm{~min}$ aggregated measurements of time-mean flow and median travel time for each 5 min on an urban arterial road "Renmin Road" in April 2015 in Changsha City, China. The region " $\mathrm{A}$ " illustrates the low demand condition and region " $\mathrm{B}$ " shows the high demand condition. It can be clearly seen in Figure 1(a) that there is no one-one correspondence relationship between travel time and flow over the whole range of traffic flow. Even in the condition of low traffic volume (region "A"), for a certain traffic flow, there is a big range of travel time corresponding to it. In the high demand condition, a large variation of travel times can be observed. Figure 1(b) illustrates travel times experienced by vehicles departing within a short time period (e.g., $1 \mathrm{~min}$ ). The difference between the maximum travel time and minimum travel time can be as large as 221 seconds even within a small departure time period of $1 \mathrm{~min}$. 


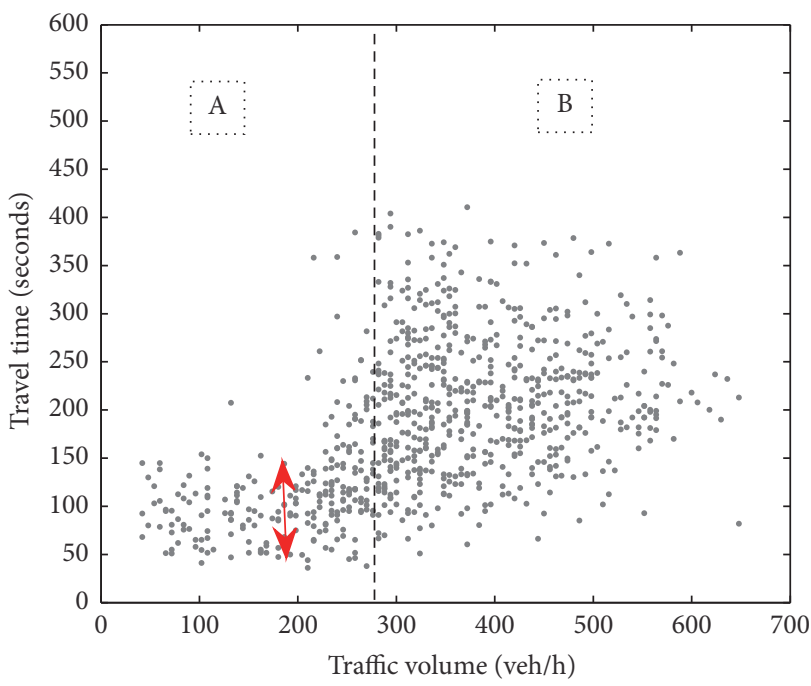

(a) Travel time versus outflow

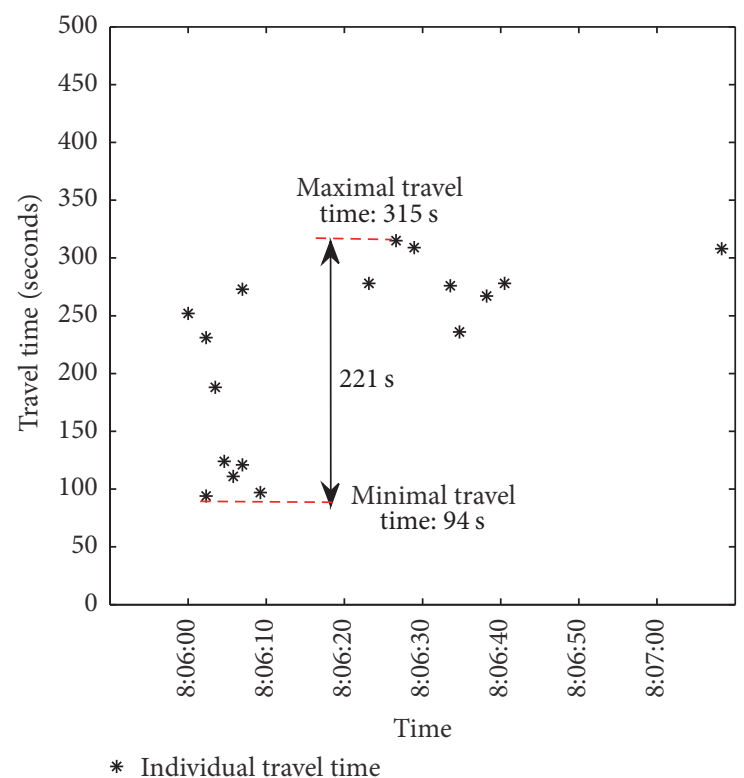

(b) Individual travel time

FIGURE 1: Variability of travel times from empirical data collected on an urban arterial road "Renmin Road" on 20 April 2015 (flow and travel time are both measured in 5 min aggregation; the outliers in travel time have been filtered out) in Changsha City, China.

2.2. Modelling Network Travel Time Distribution Using Johnson Curves. Due to a lot of uncertainties in urban transportation systems, travel times on both the link level and the route level are rather variable, which aggregately results in the variability of travel times for the network level. As shown in some empirical studies, the travel time distribution is unlikely to have a normal distribution but is heavily skewed with a long fat tail $[15,21,22]$. In this case, flexible probability densities known as Johnson curves [23] can be applied to model travel time data. The Johnson curves have been widely applied in the field of agriculture and environmental engineering and proved to be a powerful tool to model nonnormal and multimodality data $[24,25]$. One important property of Johnson curves is that parameters in the model can be estimated analytically or explicitly, which facilitate the generalization to different situations and data sets. In the following, the Johnson curves approach is discussed.

Let $X$ denote the variable of intersection-normalized travel time which is defined as the travel time trip divided by the number of intersections on the trip. Jones et al. [26] and Mahmassani et al. [19] suggested that the use of distancenormalized travel time can help exclude the source of variability coming from trip distance and only captures variability due to speed variation. This is the case with trips without intersections, when the variance of travel time depends only on the variation in speed and congestion at bottlenecks. However, on urban arterials, a major part of travel time variance comes from signalized intersections. Therefore, we propose to normalize travel time on the number of intersections for a certain trip and focus on investigating the intersectionnormalized travel time distribution for urban networks. This normalized travel time will be denoted by "travel time rate."
In the Johnson system, three types of probability density functions are used:

(1) The unbounded system $\left(\right.$ or $\left.S_{U}\right): z=\gamma+\eta \operatorname{arcsinh}((x-$ $\varepsilon) / \lambda)$

(2) The bounded system $\left(\right.$ or $\left.S_{B}\right): z=\gamma+\eta \ln ((x-\varepsilon) /(\lambda+$ $\varepsilon-x))$

(3) The lognormal system (or $\left.S_{L}\right): z=\gamma+\eta \ln ((x-\varepsilon) / \lambda$ )

where $z$ is a standard normal variable and $\gamma, \eta, \varepsilon$, and $\lambda$ are parameters of the Johnson curves. In the $S_{U}$ curves, the domain is the entire real line. By contrast, the domain of $S_{B}$ curves is defined over the open interval between $\varepsilon$ and $\varepsilon+\lambda . S_{L}$ curves can be further simplified by eliminating the parameter $\lambda$ as $z=\gamma+\eta \ln (x-\varepsilon)$. In the literature, Johnson curves were usually estimated by the method of moments $[8,27,28]$, which requires calculating four moments (mean, standard deviation, skewness, and kurtosis) of a distribution and sometimes it is not straightforward to derive these moments. Slifker and Shapiro [24] proposed a method to estimate parameters of Johnson curves based on percentiles. Their method has shown to be an easier and more reliable procedure compared with methods based on moments [25]. The procedure to estimate parameters is as follows.

Step 1. Estimate the percentiles in the travel time data corresponding to the cumulative probabilities $P_{-3 z}, P_{-z}, P_{z}$, and $P_{3 z}$ associated with four numbers $-3 z,-z, z$, and $3 z$. The value of $z$ should be chosen such that the percentiles can be reliably estimated. Slifker and Shapiro [24] suggested a choice of $z=0.524$, which provides reliable estimates of four percentiles and works well for a variety of data sets. 
Step 2. Estimate the percentile travel times at $x_{-3 z}, x_{-z}, x_{z}$, and $x_{3 z}$ based on the probabilities $P_{-3 z}, P_{-z}, P_{z}$, and $P_{3 z}$ and calculate the parameters $m, n$, and $p$ as

$$
\begin{aligned}
& m=x_{3 z}-x_{z}, \\
& n=x_{-z}-x_{-3 z}, \\
& p=x_{z}-x_{-z} .
\end{aligned}
$$

Slifker and Shapiro [24] showed that the expression $m n / p^{2}$ can be used to determine the appropriate Johnson curve. The Johnson $S_{U}$ corresponds to $m n / p^{2}>1$, the Johnson $S_{B}$ corresponds to $m n / p^{2}<1$, and Johnson $S_{L}$ corresponds to $m n / p^{2}=1$. It should be noted that when using the value of $m n / p^{2}$ to select among Johnson curve types, $S_{U}$ and $S_{B}$ predominate since only in rare cases $m n / p^{2}$ will be exactly equal to 1 .

Step 3. Estimate the Johnson curve parameters $\gamma, \eta, \varepsilon$, and $\lambda$. For the detailed calculation of these parameters, readers can refer to literature $[24,25]$.

Based on the above estimated parameters, travel time rate distributions using the Johnson curves can be derived straightforwardly. It is worthwhile noting that the circumstances in which $m n / p^{2}$ exactly equals 1 are rather rare. Therefore, $S_{U}$ and $S_{B}$ are more frequently chosen when the value of $\mathrm{mn} / \mathrm{p}^{2}$ is used to determine the type of Johnson curves. However, $m n / p^{2}$ could be less than or larger than 1 when samples are extracted from an $S_{L}$ distribution in some data sets [29]. In this case, examination of the third (skewness) and fourth (kurtosis) moments of data in relation to the moment plane could help determine the proper type of Johnson curves when $m n / p^{2}$ is close to 1 . For instance, as suggested by Mage [29], if the (third and fourth) moments lie within the $S_{B}$ region and $m n / p^{2}$ is larger than 1 , the $S_{L}$ distribution is an appropriate choice.

\subsection{Illustration with Field ANPR Data}

2.3.1. Study Area and Data Description. In order to investigate how the Johnson curve system performs in terms of describing network travel time distribution (variability), we selected a study network located in the CBD of Changsha City, China. Figure 2 shows the selected network with 73 signalized intersections and 21 intersections with ANPR cameras. The data were collected for three days from 20 April 2015 to 22 April 2015. The time period of analysis considered in this study is between 7:00 a.m. and 7:00 p.m. Each day was divided into $30 \mathrm{~min}$ time intervals. OD travel times between intersections with ANPR can be estimated from camera records. Outliers such as double counting and mismatching were eliminated from the data set. Apart from these outliers, by comparing travel times between vehicles consecutively passing the intersection, abnormal link travel times between two adjacent cameras (e.g., parking along the road for a coffee) were identified and eliminated as well. The process can be described as follows.
Vehicles $i-1, i, i+1, \ldots, n$ sequentially pass intersections $j, j+1, \ldots, m$. Let us denote $\vartheta_{i}^{j}$ as the time instant when vehicle $i$ passes the upstream intersection $j, \vartheta_{i-1}^{j}$ as the time instant when the immediate preceding vehicle $i-1$ passes the upstream intersection $j, \vartheta_{i+1}^{j}$ as the time instant when the immediate following vehicle $i+1$ passes the upstream intersection $j, \vartheta_{i-1}^{j+1}$ as the time instant when vehicle $i-1$ passes the adjacent downstream intersection $j+1, \vartheta_{i}^{j+1}$ as the time instant when vehicle $i$ passes the adjacent downstream intersection $j+1$, and $\vartheta_{i+1}^{j+1}$ as the time instant when vehicle $i+1$ passes the adjacent downstream intersection $j+1$; then the link travel time between intersections $j$ and $j+1$ for vehicles $i-1, i$, and $i+1$ can be calculated as

$$
\begin{aligned}
& \tau_{i-1}^{j \rightarrow j+1}=\vartheta_{i-1}^{j+1}-\vartheta_{i-1}^{j}, \\
& \tau_{i}^{j \rightarrow j+1}=\vartheta_{i}^{j+1}-\vartheta_{i}^{j}, \\
& \tau_{i+1}^{j \rightarrow j+1}=\vartheta_{i+1}^{j+1}-\vartheta_{i+1}^{j} .
\end{aligned}
$$

The link travel time differences between vehicle $i-1$ and $i$ and vehicle $i$ and $i+1$ are calculated as

$$
\begin{aligned}
& \Delta \tau^{-}=\tau_{i}^{j \rightarrow j+1}-\tau_{i-1}^{j \rightarrow j+1}, \\
& \Delta \tau^{+}=\tau_{i}^{j \rightarrow j+1}-\tau_{i+1}^{j \rightarrow j+1} .
\end{aligned}
$$

If $\Delta \tau^{-}>\delta$ and $\Delta \tau^{+}>\delta$, vehicle $i$ is considered as "abnormal" vehicle whose travel time is much longer compared with "normal" preceding vehicle $i-1$ and following vehicle $i+1$. Then the link travel time of vehicle $i$ is eliminated, where $\delta$ is the predefined threshold (the average cycle time of the intersections is about $3 \mathrm{~min}$; therefore, " $3 \mathrm{~min}$ " is chosen in our case).

The vehicle travel time rates were then calculated with individual travel times divided by their travel distance.

2.3.2. Results. We calculated the value of parameters $\gamma, \eta, \varepsilon$, and $\lambda$ in the Johnson curve system according to the steps described in the previous subsection. The value of $m n / p^{2}$ is smaller than 1 for all cases, which indicates that the Johnson $S_{B}$ curves are appropriate to describe travel time rate distributions for our selected network.

Figures 3(a)-3(c) illustrate the comparison between the network travel time rate distributions estimated from ANPR data and the Johnson fitted and lognormal fitted curves during peak time period of 7:00-7:30 a.m. for three days. The Johnson curves show a better fit to the ANPR travel time data compared with the lognormal fit. Similar results can be identified in Figures 3(d)-3(f), where ANPR travel time rate distributions are presented along with Johnson and lognormal fitted probability curves during off-peak period of 10:00-10:30 a.m. We further applied the one-sample Kolmogorov-Smirnov (KS) test with four time periods each day (in total, 12 time periods). Table 1 presents the $K-S$ test results. From Table 1, it can be concluded that the null hypothesis that the travel time rates follow the Johnson curve (SB) distribution cannot be rejected for most of the time periods 


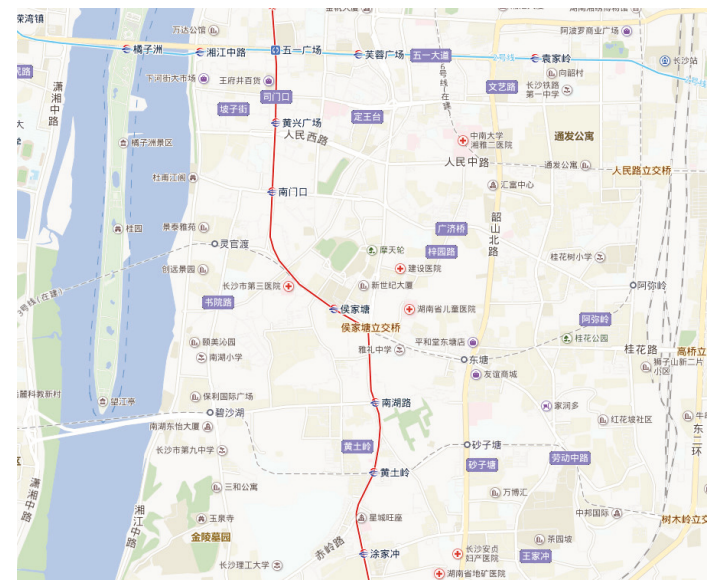

(a) Changsha $\mathrm{CBD}$ area

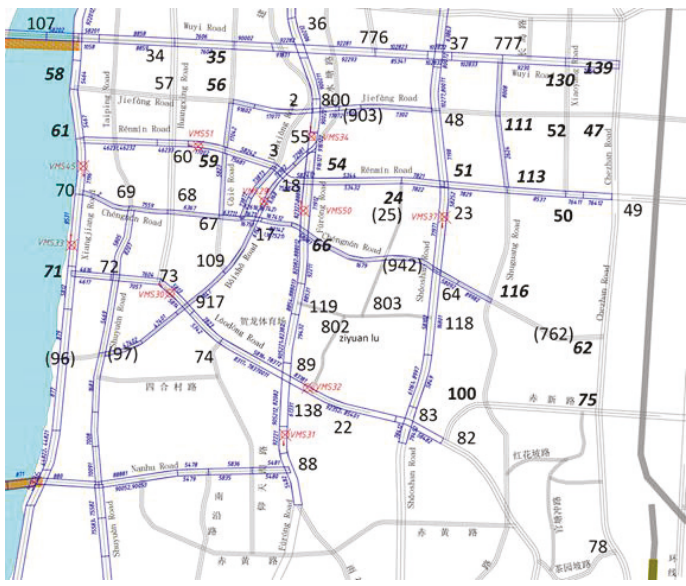

(b) Intersections with ANPR cameras

FIgure 2: The selected network in Changsha City, China.

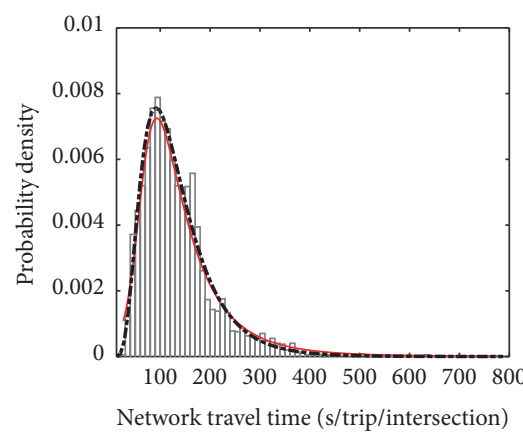

- ANPR data

— Lognormal

---. Johnson curve

(a) 2015-04-20 (7:00-7:30 a.m.)

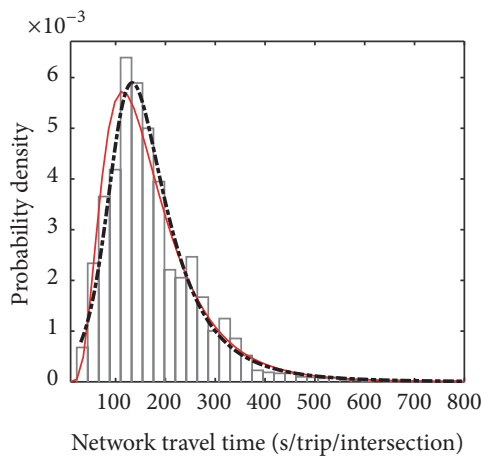

- ANPR data

— Lognormal

---- Johnson curve

(d) 2015-04-20 (10:00-10:30 a.m.)

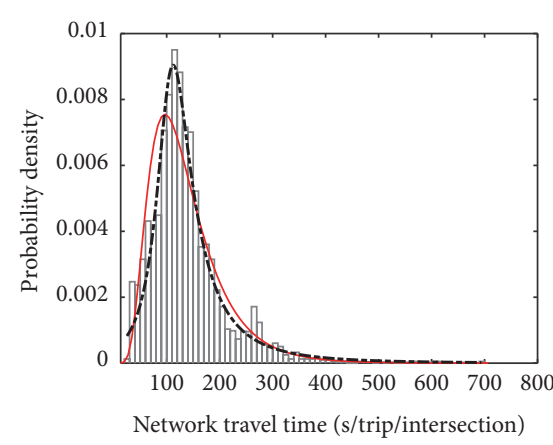

- ANPR data

— Lognormal

---- Johnson curve

(b) 2015-04-21 (7:00-7:30 a.m.)

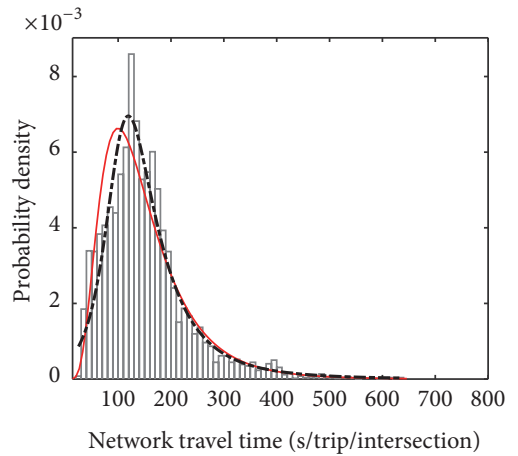

- ANPR data

— Lognormal

---- Johnson curve

(e) 2015-04-21 (10:00-10:30 a.m.)

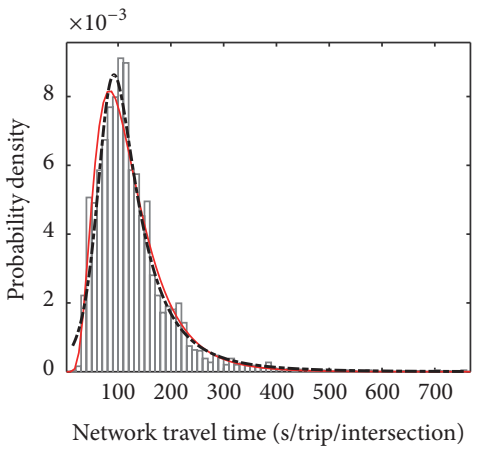

- ANPR data

— Lognormal

---- Johnson curve

(c) 2015-04-22 (7:00-7:30 a.m.)

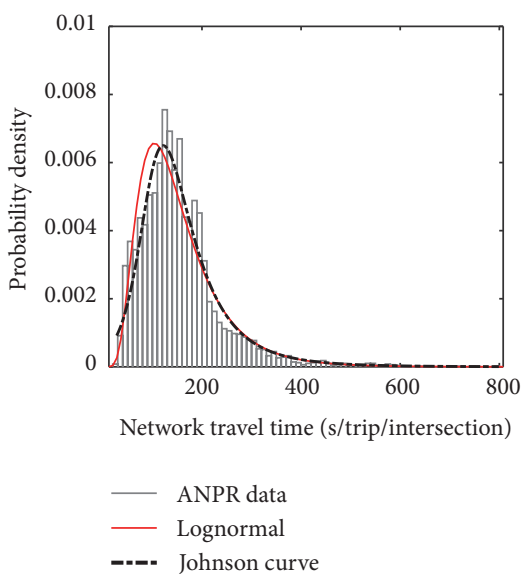

(f) 2015-04-22 (10:00-10:30 a.m.)

FIGURE 3: Network travel time rate distribution from ANPR data and fitted density curves at morning peak-hour period ((a)-(c)) and morning off-peak period ((d)-(f)). 
TABle 1: $P$ value of Kolmogorov-Smirnov (KS) test with significance level of 0.05 .

\begin{tabular}{|c|c|c|c|c|}
\hline \multicolumn{5}{|c|}{$P$ value } \\
\hline Fitted distributions & Time period & Day 1 & Day 2 & Day 3 \\
\hline \multirow{4}{*}{ Johnson curve } & 7:00 a.m.-7:30 a.m. & 0.456 & 0.334 & 0.284 \\
\hline & 10:00 a.m. $-10: 30$ a.m. & 0.312 & 0.265 & 0.202 \\
\hline & 3:00 p.m.- 3:30 p.m. & 0.258 & 0.168 & 0.126 \\
\hline & 5:00 PM-5:30 p.m. & 0.359 & 0.032 & 0.243 \\
\hline \multirow{4}{*}{ Lognormal fit } & 7:00 a.m.-7:30 a.m. & 0.392 & 0.036 & 0.122 \\
\hline & 10:00 a.m.-10:30 a.m. & 0.087 & 0.032 & 0.071 \\
\hline & 3:00 p.m.- 3:30 p.m. & 0.036 & 0.073 & 0.048 \\
\hline & 5:00 p.m. $-5: 30$ p.m. & 0.033 & 0.059 & 0.076 \\
\hline
\end{tabular}

Day 1: 2015-04-20; day 2: 2015-04-21; day 3: 2015-04-22.

TABLE 2: AIC values for the Lognormal model and the Johnson-curve model.

\begin{tabular}{lccrr}
\hline & & AIC value & & \\
Fitted distributions & Time period & Day 1 & Day 2 & 16988 \\
\hline \multirow{2}{*}{ Johnson curve } & 7:00 a.m.-7:30 a.m. & 15823 & 167154 \\
\hline \multirow{2}{*}{ Lognormal } & $10: 00$ a.m.-10:30 a.m. & 16343 & 16772 & 16952 \\
& $7: 00$ a.m.-7:30 a.m. & 16128 & 18214 & 17993 \\
& $10: 00$ a.m.-10:30 a.m. & 16766 & 17525 & 17629 \\
\hline
\end{tabular}

(except time periods 5:00 p.m.-5:30 p.m. on day 2015-0421 ) at the significance level of $5 \%$ since the $P$ value is larger than 0.05 . The better fitting performance of the Johnson curve model is due to the fact that the Johnson system allows describing a wide spectrum of traffic conditions as usually existing in the urban traffic system. The Johnson model can flexibly adapt its shape to the observed travel time data. We calculated the Akaike information criterion (AIC) for both the Johnson curve model and the lognormal model as well. AIC is a measure of the relative quality of statistical models for a given set of data. It is defined as

$$
\mathrm{AIC}=2 * k-2 \ln (L),
$$

where $k$ is the number of parameters in the model; $L$ is the maximized value of the likelihood function for the model. From (4), it can be seen that AIC rewards goodness of fit (as assessed by the likelihood function), but it also includes a penalty that is an increasing function of the number of estimated parameters.

Given a set of candidate models for the data, the preferred model is the one with the minimum AIC value. For more detailed information about AIC, readers can refer to Akaike's work [30]. Table 2 shows the AIC values for both models in different time periods. The lower AIC values of the Johnson curve model further confirm that the Johnson curve model outperforms the lognormal distribution model.

Besides, as can be seen from Figure 3, network travel time distributions are wide and skewed with a long fat right tail during different time periods, which suggest that the network travel time rate is rather variable (unreliable). In the following section, we will look at network travel time reliability measures and how these measures vary under different traffic conditions.

\section{Network Travel Time Reliability Metrics and Their Connection with Network Traffic Characteristics}

3.1. Network Travel Time Reliability Measures. Based on travel time distributions as proposed in the previous section, any travel time reliability metrics (e.g., standard deviation, percentiles, skewness, and buffer index) can be derived. In the past decades, travel time reliability metrics are commonly used to quantify link or route travel time reliability, whereas very little research has been dedicated to investigate the network travel time reliability. In this section, we investigate the network travel time reliability (in terms of intersectionweighted standard deviation and intersection-weighted skewness of travel time rate) and its connection with network traffic characteristics under different traffic conditions.

A method that is frequently used in literature to characterize traffic states in a network is the macroscopic fundamental diagram (MFD) [31-33]. In the MFD, the traffic state is characterized by production (sum of distance-weighted traffic flow) and accumulation (sum of distance-weighted traffic density). Let us denote the length of a link $j$ by $l_{j}$, the outflow of link $j$ by $q_{j}$, the traffic density (vehicles per length of the road) of link $j$ by $k_{j}$, and the total number of links in the network by $n$. Then, the weighted average network density during time interval $\Delta t$ is defined as

$$
\bar{k}(\Delta t)=\frac{\sum_{j=1}^{n} l_{j} k_{j}(\Delta t)}{\sum_{j=1}^{n} l_{j}} .
$$

Since we use ANPR data, the traffic density cannot be derived directly. We calculate the traffic density from the total vehicle travel time on link $j$ and the link length $l_{j}$. We use the methodology proposed by Edie [34] applied on single links, 


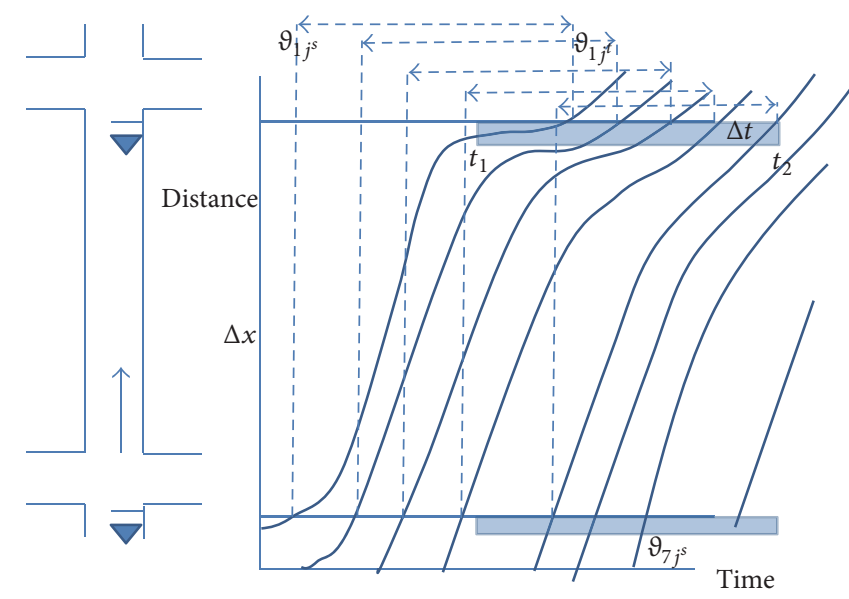

FIgURE 4: Trajectories at the boundary of the time frame.

based on trajectory analysis [35]. The average traffic density of link $j k_{j}$ during time interval $\Delta t$ is estimated as the total time spent on the link during the time interval.

There is a methodological problem in the choice of the frame in space and time for the definition of the traffic density. The time the vehicle $i$ spent on the link $j t_{i j}$ for the first few vehicles passing the end of the link after $t_{1}$ is not entirely spent during the time period $\Delta t$ as shown in Figure 4 . The situation is represented in a rather extreme way to show the problem. The first four vehicles registered by the camera have a travel time which has not been spent completely in the time frame $\Delta t$. The next four vehicles are not registered in time frame $\Delta t$ although they spend a part of the time in the space-time frame $\Delta x \cdot \Delta t$. Although the detailed trajectories are not known, we can apply the Three-Dimensional Trajectory method (Saberi et al. [35]). To be accurate we have to correct the travel time of the first vehicles passing in $\Delta t$, that is, the vehicles for which the travel time is longer than the time in $\Delta t$ at which they are registered. Similarly, we have to add at the end of the timeframe the times spend by the vehicles that entered $\Delta x$ but did not reach the end of the road section within the timeframe.

The travel time of vehicle $i$ on link $j$ is given by the difference in passing time at the stop line of the link $j \vartheta_{i j}^{t}$ and the link $j-1 \vartheta_{i j}^{s}$ as $t t_{i j}=\vartheta_{i j}^{s}-\vartheta_{i j}^{t}$. The part of the travel time that should be allocated to the time interval $\Delta t$ is calculated as

$$
t t_{i j}^{\prime}=\min \left\{t t_{i j}, \vartheta_{i j}^{t}-t_{1}\right\}, \quad t_{1} \leq \vartheta_{i j}^{t} \leq t_{2} .
$$

In an analogue way, the vehicles that are not counted at the end of the time frame can be detected in the next time frame and partly allocated to $\Delta t$. The travel time that has to be added to the time spent in the period $\Delta t$ is calculated as

$$
t t_{i j}^{\prime}=t_{2}-\vartheta_{i j}^{s}, \quad \text { for } \vartheta_{i j}^{s}<t_{2}, \vartheta_{i j}^{t}>t_{2} .
$$

In practice, if the time intervals $\Delta t$ are chosen longer than the travel time on the link the boundary problem gives only a marginal influence of the calculation of the time spent.
Furthermore, $\Delta t$ is chosen long enough to have little influence of the signal cycle time (which was not a common cycle time for all intersections). This avoids the time-space window problems as discussed by Courbon and Leclercq [36]

$$
k_{j}(\Delta t)=\frac{\sum_{i=1}^{m}\left(t t_{i j}^{\prime} / l_{j}\right)}{\Delta t} \quad\left(\Delta t=t_{2}-t_{1}\right)
$$

where $t t_{i j}^{\prime}$ is the corrected travel time of vehicle $i$ on link $j$ passing the stop line at the end of link $j$ between $t_{1}$ and $t_{2}$; $m$ is the total number of vehicles leaving link $j$ during time interval $\Delta t$ united with the vehicles that entered the link $j$ in $\Delta t ; n$ is the total number of links in the network; $t_{1}$ and $t_{2}$ are the start and end of the time interval.

The weighted (average) network flow during time interval $\Delta t$ is defined as

$$
\bar{q}(\Delta t)=\frac{\sum_{j=1}^{n} l_{j} q_{j}(\Delta t)}{\sum_{j=1}^{n} l_{j}},
$$

where $q_{j}(\Delta t)$ is determined as the number of vehicles passing the end of link $j$ during $\Delta t$. Apart from the network flow and density, three reliability related quantities which are intersection-weighted mean travel time rate, intersection-weighted standard deviation of travel time rate, and intersection-weighted skewness of travel time rate distributions are used in this study.

As we proposed in the previous section, the travel time rate of trip $i$ can be calculated as the trip travel time $t_{i}$ divided by the number of intersections $n_{i}$ during this trip:

$$
\tau_{i}=\frac{t_{i}}{n_{i}} .
$$

The intersection-weighted mean travel time rate during time interval $\Delta t$ is defined as

$$
\bar{T}(\Delta t)=\frac{\sum_{i=1}^{N} n_{i} \tau_{i}(\Delta t)}{\sum_{i=1}^{N} n_{i}},
$$

where $n_{i}$ is the number of intersections during trip $i, \tau_{i}$ is the travel time rate of trip $i$, and $N$ is the total number of trips observed in the network during time period $\Delta t$.

The intersection-weighted standard deviation of travel time rate during time interval $\Delta t$ is defined as

$$
\bar{\sigma}(\Delta t)=\sqrt{\frac{\sum_{i=1}^{N} n_{i}\left(\tau_{i}(\Delta t)-\bar{T}(\Delta t)\right)^{2}}{\sum_{i=1}^{N} n_{i}}} .
$$

Skewness is a measure of the asymmetry of the probability distribution of a random variable about its mean. In terms of reliability, the more skewed the travel time distribution is, the less reliable travel times become. If the value of skewness is zero, the travel time is normally distributed. If the value of skewness is larger than zero, the travel time distribution is right-skewed with a longer tail to the right. Otherwise, the travel time distribution is left-skewed with a longer tail to the 


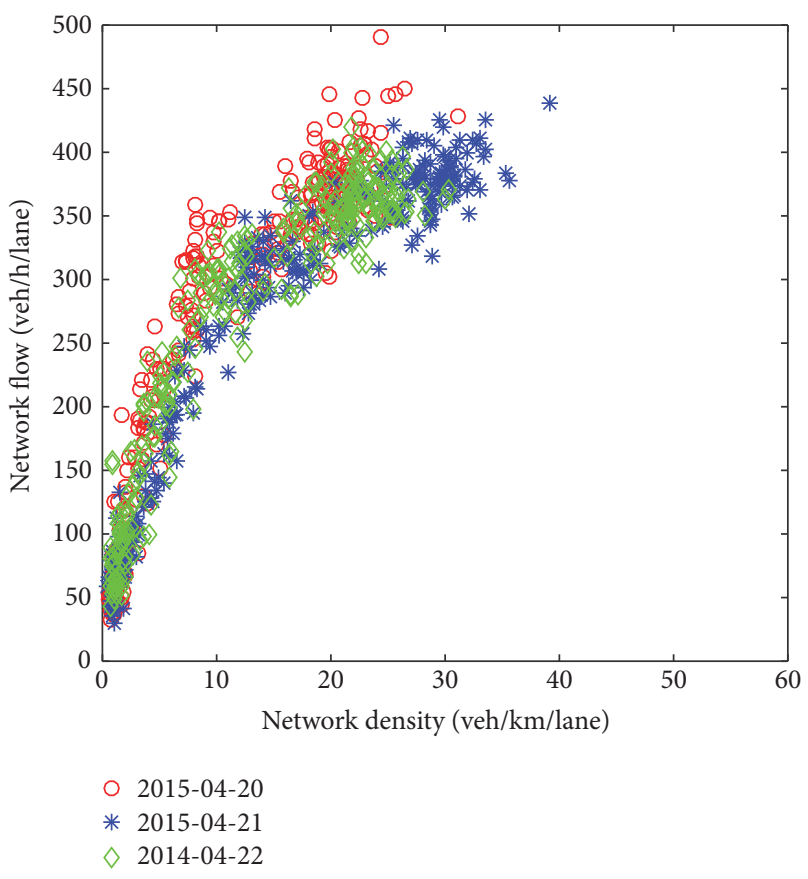

(a) Time interval of $5 \mathrm{~min}$

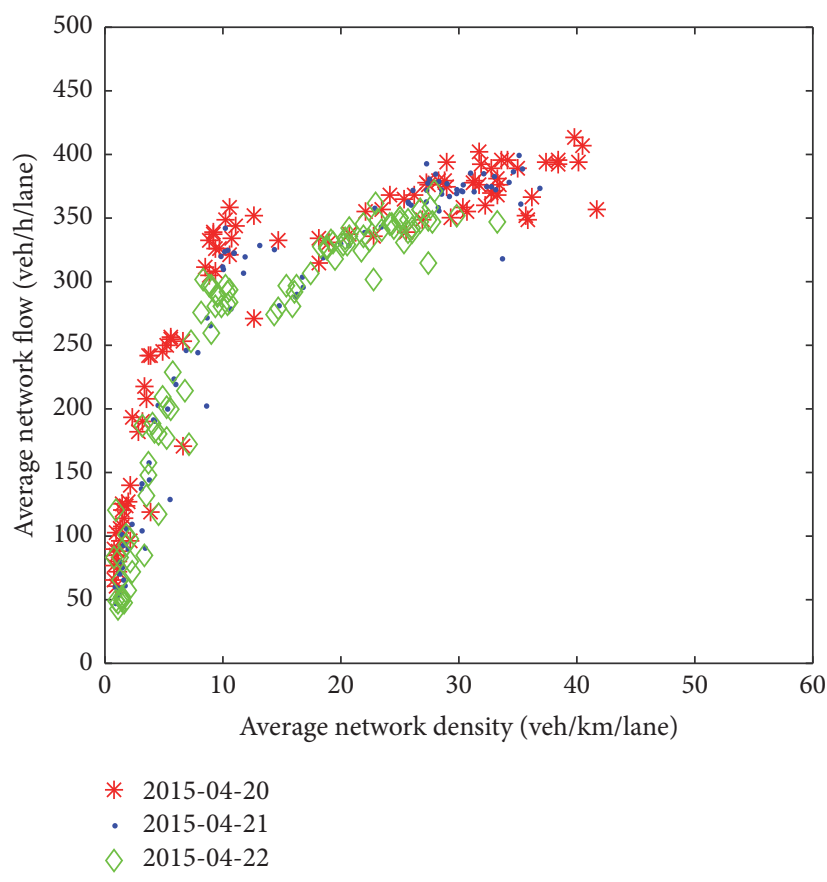

(b) Time interval of $15 \mathrm{~min}$

FIGURE 5: Network flow-density diagram for the study area in Changsha City (7:00 a.m.-7:00 p.m.).

left. The weighted skewness of travel time rate for a network during time interval $\Delta t$ is defined as

$$
\bar{S}(\Delta t)=E\left[\frac{\sum_{i=1}^{N} n_{i}\left(\left(\tau_{i}(\Delta t)-\mu(\Delta t)\right) / \sigma(\Delta t)\right)^{3}}{\sum_{i=1}^{N} n_{i}}\right],
$$

where $\mu(\Delta t)$ is the mean travel time rate during time interval $\Delta t ; \sigma(\Delta t)$ is the standard deviation of travel time rate during time interval $\Delta t$.

3.2. Case Study with Field ANPR Data. The selected study area consisting of 54 links is shown in Figure 2 in the previous section. Again we use the three-day (between 7:00 a.m. and 7:00 p.m. from 20 April until 22 April 2015) ANPR data from Changsha City, China. The traffic flow can be derived directly since each ANPR camera records the plates number of all vehicles on lanes for straight-on and left turning movements passing the stop line. The traffic density however cannot be obtained directly from ANPR data. We apply (6) to estimate traffic density for each link. Figure 5 illustrates the macroscopic fundamental diagram for the selected CBD network based on three days' observations with time intervals of $5 \mathrm{~min}$ (Figure 5(a)) and $15 \mathrm{~min}$ (Figure 5(b)), respectively. This macroscopic fundamental diagram reveals that the observed maximum weighted traffic volume in this network is around $400 \mathrm{veh} / \mathrm{h} / \mathrm{lane}$ for the weighted traffic density around $30 \mathrm{veh} / \mathrm{km} / \mathrm{lane}$. No gridlock happened in this region from the observed data. That does not mean that the network operates below capacity, but it is an indication that the traffic police actively takes measures to reduce inflow and increase outflow on links at the moment that spillback threatens to occur.
Of course, this form of active traffic management has an influence on travel times and travel time reliability.

Figure 6 illustrates the relation between the weighted average travel time rate and the weighted standard deviation of travel time rate for every 5 min. Figures $6(a)-6(c)$ are intersection-weighted travel times and Figures 6(d)-6(f) are distance-weighted travel times. A clear linear trend can be observed with both intersection-normalized and distancenormalized approaches, though the travel time data appear to be more scattered with a slightly lower correlation coefficient $\left(R^{2}=0.7167\right.$ and 0.5938$)$ on 21 April. Higher correlations can be observed with intersection-weighted approach compared with the distance-weighted method for all three days' travel times. The magnitude of the slope which reflects how much the weighted standard deviation will increase when the weighted average travel time rate increases is between 0.46 and 0.65 for intersection-weighted approach and between 0.35 and 0.62 for distance-weighted approach within these three days' observations. The larger this value is, the more vulnerable to congestion induced reliability deterioration the network becomes.

Figure 7 shows the relation between the weighted average travel time rate and the network density, where the upper three figures (Figures $7(a)-7(\mathrm{c})$ ) are intersection-weighted travel time rates and the lower three ones (Figures $7(d)-7(f)$ ) are distance-weighted travel time rates. A relatively strong positive correlation exists between the weighted average travel time rate and the network density for all three days with $R^{2}>0.55$ for intersection-weighted method and $R^{2}>0.48$ for distance-weighted approach. The comparison between the intersection-weighted method and the distance-normalized 


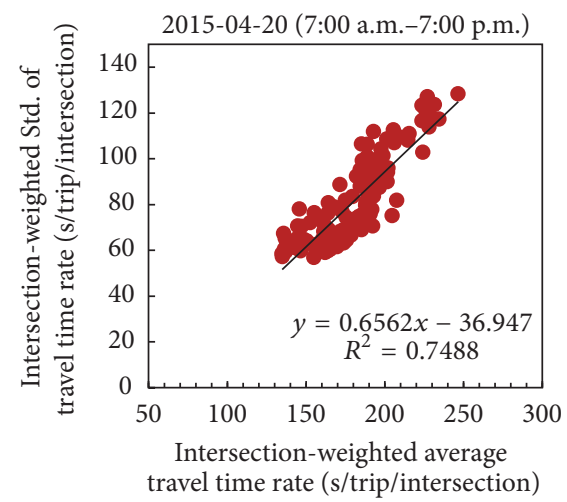

(a)

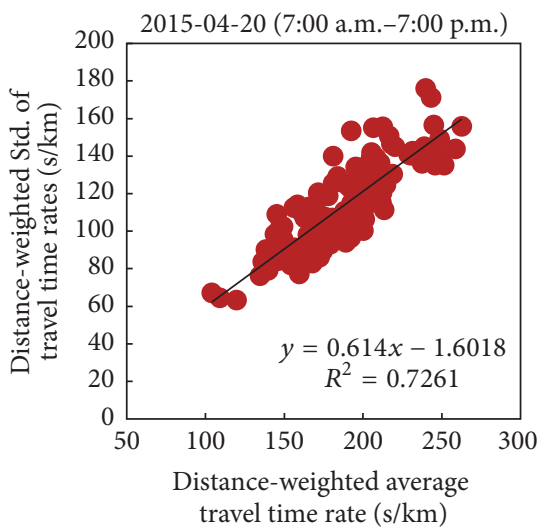

(d)

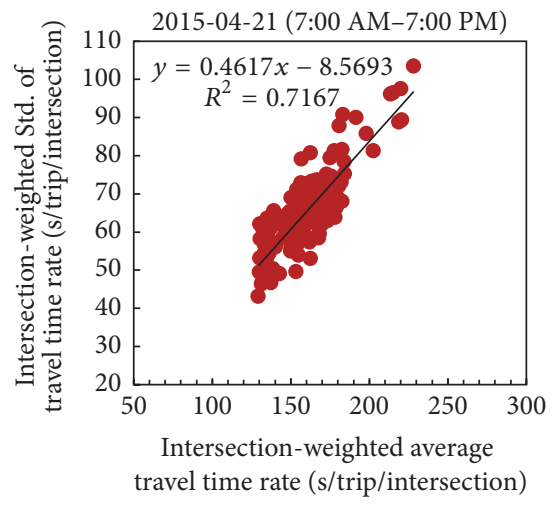

(b)

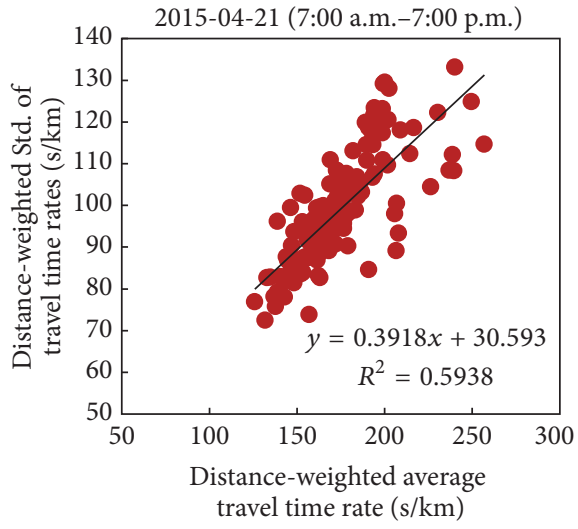

(e)

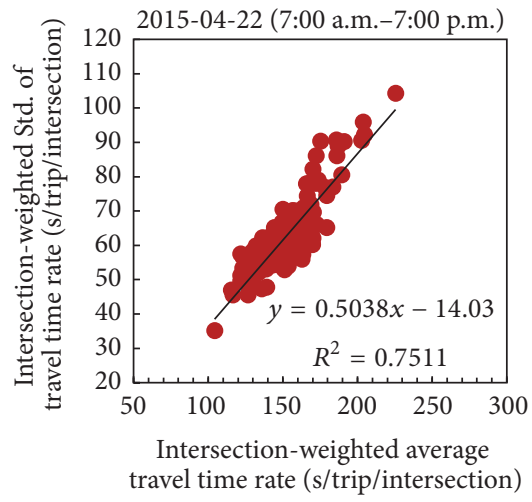

(c)

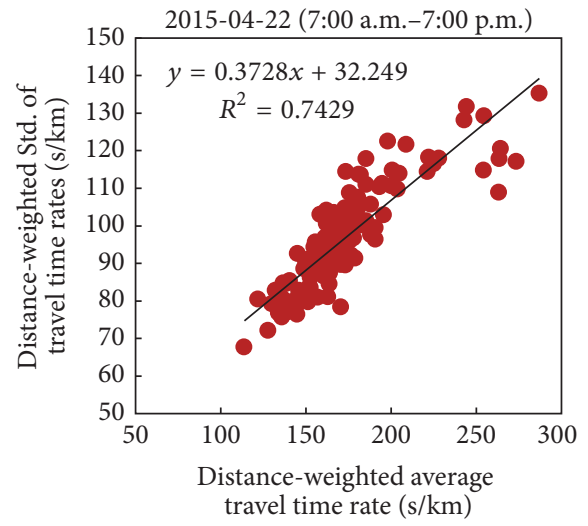

(f)

FIGURE 6: Weighted average travel time rate versus weighted standard deviation of travel time rate.

method illustrates that closer correlations can be observed with the former than the later. Lower correlation coefficients can be observed for the weighted standard deviation versus the network density as shown in Figure 8. Nevertheless, the positive linear trend still exists, which indicates that the travel time variability increases when the network density increases. This is consistent with the argument in literature that the network travel time variability has a linear relation with the network density with simulated data [37].

Apart from investigating network travel time variability in terms of the standard deviation, the intersection-weighted skewness of ANPR travel time data is calculated as well according to (15). Figure 9 shows the relation between the weighted skewness and the network density. There is no strong correlation between the network density and skewness, though a weak trend, indicating that the skewness decreases with the increase of network density, can be observed. Nevertheless, we can observe that most of the values of skewness between 7:00 a.m. and 7:00 p.m. are larger than zero, which indicates that the travel time distribution is right-skewed with a longer right tail. This is consistent with travel time distributions derived in Section 2 with Johnson curves.
The relations between travel time reliability and network flow are illustrated in Figure 10. It is observed that the relationship between travel time reliability (e.g., the intersectionweighted standard deviation of travel time rate) and network flow rate is not monotonic anymore. The intersectionweighted standard deviation of travel time rate increases with network flow rate in low traffic demand condition until the maximum flow rate, beyond which the network flow rate drops while the standard deviation continues to increase. The results shown in Figure 10 are quite consistent with those derived from simulated data in [37].

\section{Discussion and Conclusions}

This paper proposes a network-level travel time distribution model by applying a flexible system of Johnson curves. The proposed model is able to describe a variety of travel time distributions with properly estimating model parameters based on the observed percentiles in the travel time data. We validate the performance of the proposed model using ANPR data collected in the CBD area of Changsha City, China. The results indicate that the travel time data can be better modelled with the proposed model than the widely used lognormal model in the transportation field. The reason behind 


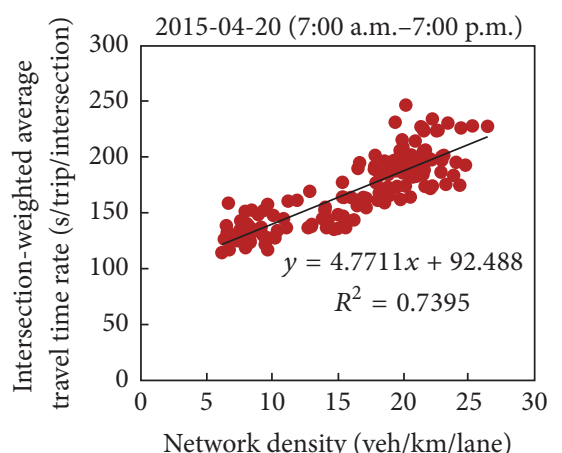

(a)

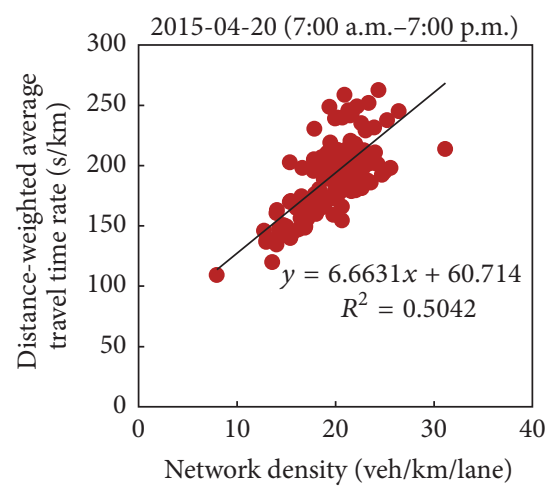

(d)

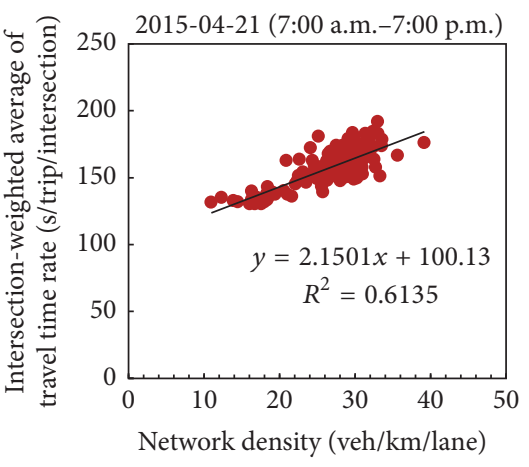

(b)

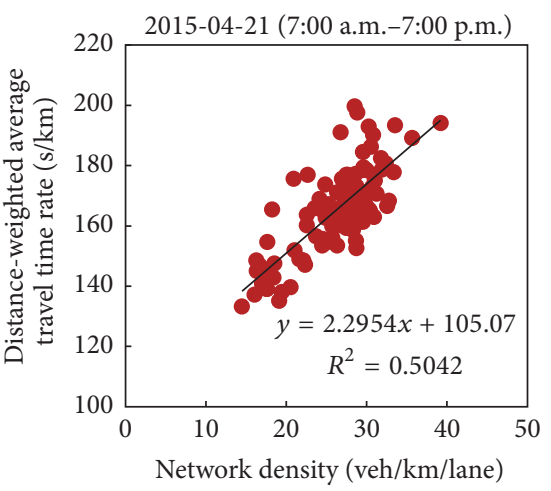

(e)

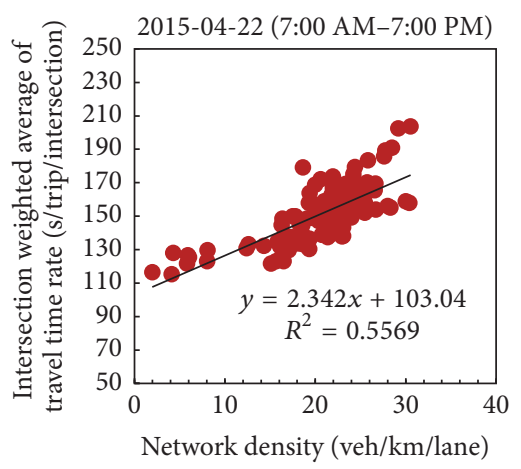

(c)

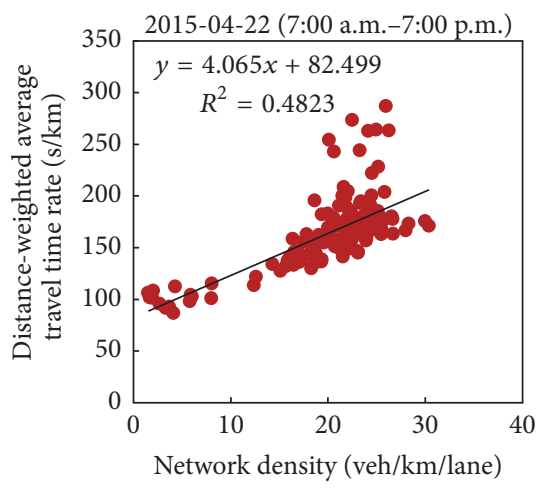

(f)

FIGURE 7: Weighted average travel time rate versus network density.

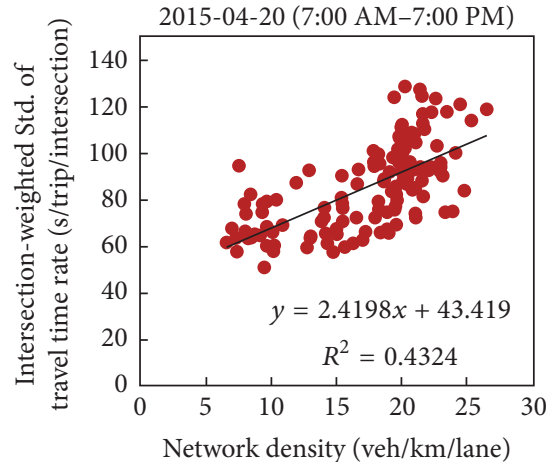

(a)

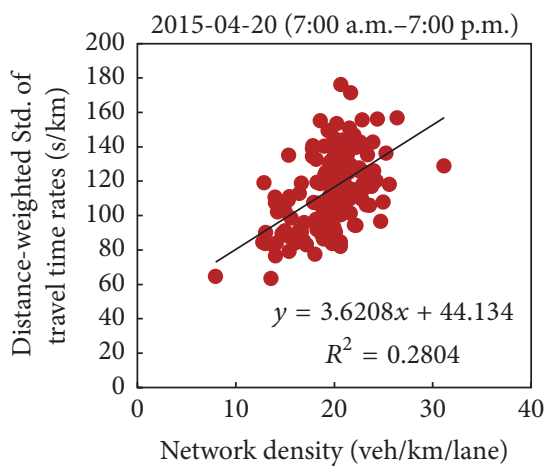

(d)

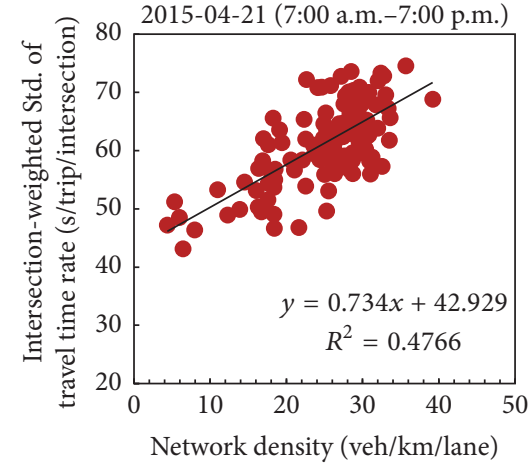

(b)

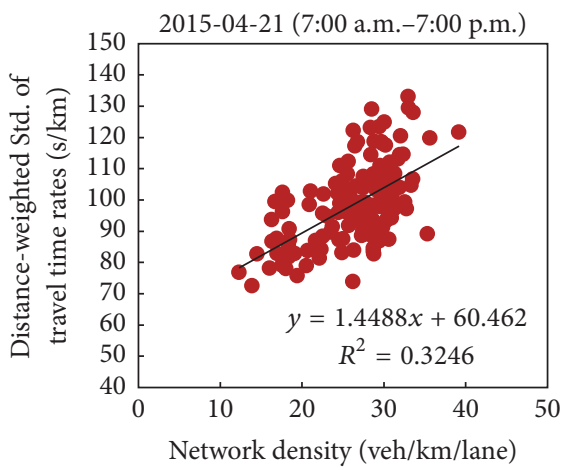

(e)

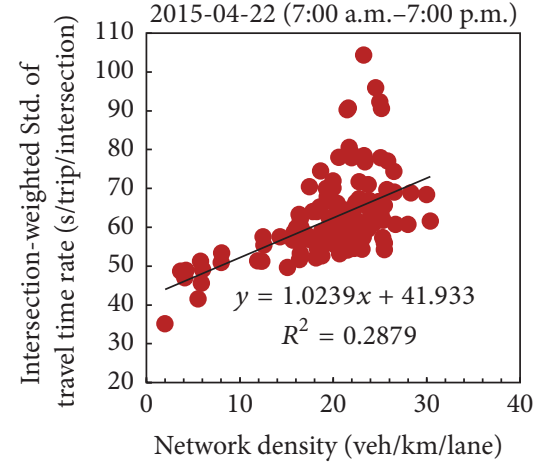

(c)

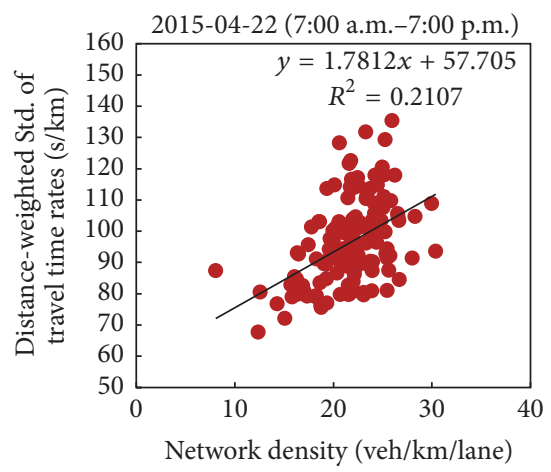

(f)

FIGURE 8: Weighted standard deviation of travel time rate versus network density. 


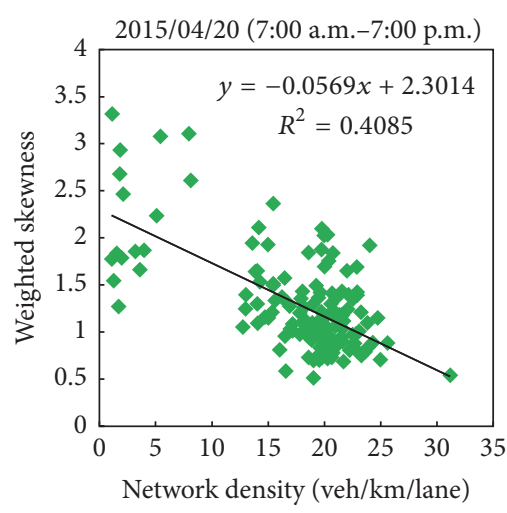

(a)

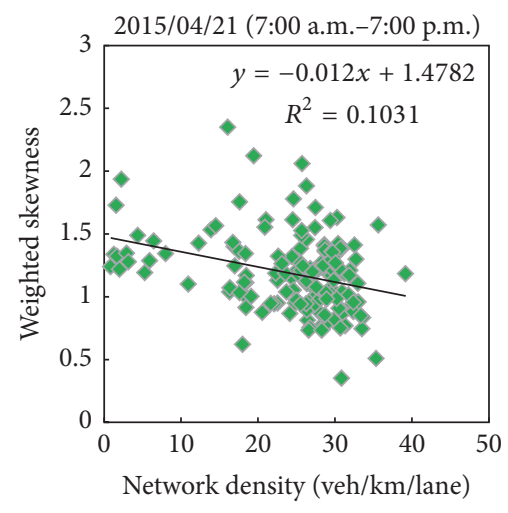

(b)

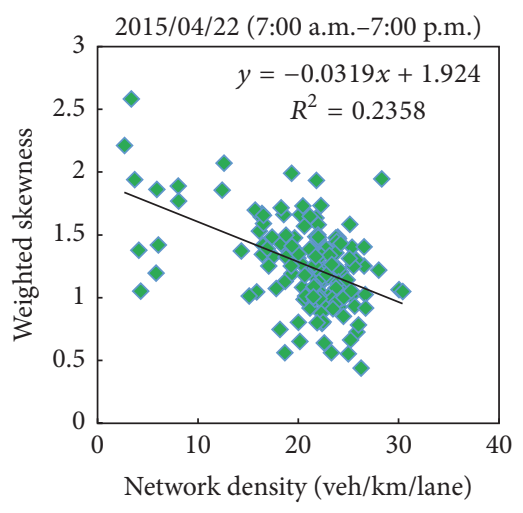

(c)

FIGURE 9: Intersection-weighted skewness versus network density.

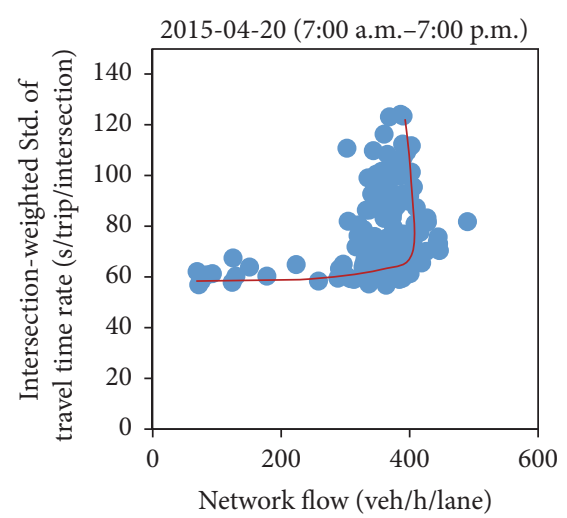

(a)

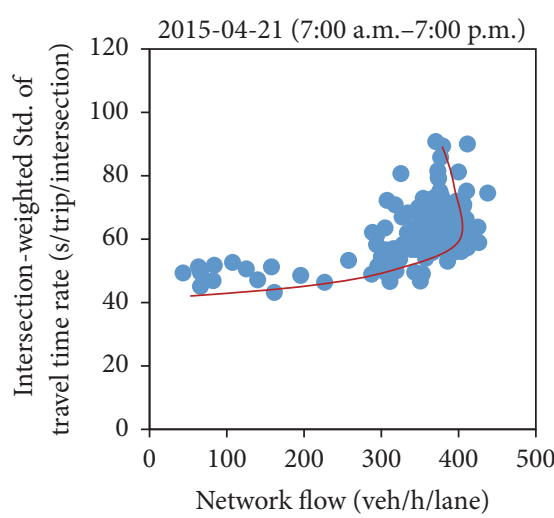

(b)

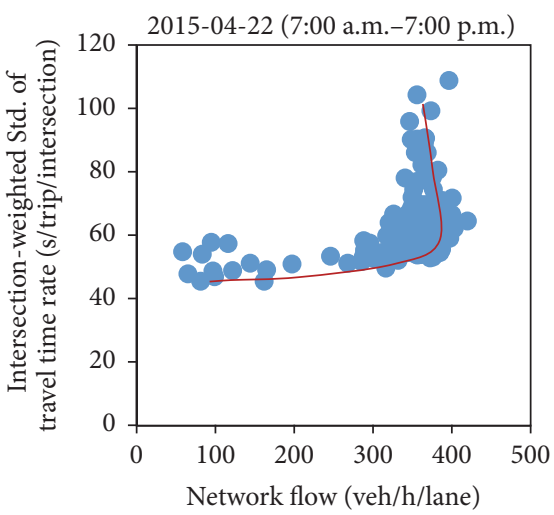

(c)

FiguRE 10: Intersection-weighted standard deviation of travel time rate versus network flow.

is that the Johnson curve model is able to better capture the skewness in the data set with parameters describing the tail of the distribution (e.g., with the third and fourth moments or four percentile points), while in the lognormal distribution, only the mean and standard deviation of the logarithm are used to characterize the distribution of the data set.

We further investigated the network travel time reliability measures and their connection with network traffic characteristics in terms of network density and network flow, which are known as the macroscopic fundamental diagram. Three network travel time reliability measures which are the weighted average travel time rate, the weighted standard deviation of travel time rate, and the weighted skewness of travel time distributions are discussed, and the relation between these reliability measures and the network density is illustrated using the field ANPR data. The weighting is done with respect to both the number of intersections on a trip and the trip distance. Results show that the weighted average travel time rate has a positive linear relation with the weighted standard deviation of travel time rate for different time periods under time-varying demand. A positive linear trend can be clearly observed between the weighted standard deviation (or the weighted average travel time rate) and the network density. The correlations between reliability measures and network states are higher with the intersection-weighted approach than those using the distance-weighted approach. The investigation of the relation between the weighted skewness and the network density shows that there is a weak negative correlation between the former and the latter based on the available ANPR data. The value of skewness is larger than 0 for most observation periods between 7:00 a.m. and 7:00 p.m., which indicates that travel time distributions are right-skewed with a longer tail to the right.

The findings in this paper provide some insights into the network travel time reliability. The empirical relation between the network travel time reliability and network traffic characteristics (e.g., network density) can be possibly applied to assess the traffic management and control measures for reliability purposes; for example, by applying the relations between network flow and network density, as well as network reliability (network standard deviation) and network density, network control strategies can be optimized to improve network reliability while maintaining required network throughput.

\section{Conflicts of Interest}

The authors declare that they have no conflicts of interest. 


\section{Acknowledgments}

This work is supported by the National Science Foundation of China (NSFC) under Projects 51308475, 61673321, and 71671147, the Fundamental Research Funds for the Central Universities under Contract 2682016CY07, Chengdu Science and Technology Commission under Contract 2015-RK0000190-ZF, and Changsha Science and Technology Commission under Projects K1001010-11 and K1106004-11.

\section{References}

[1] F. Zheng, H. van Zuylen, and Y. Pu, "Model for predicting distribution of link travel times for urban signalized roads," Transportation Research Record: Journal of the Transportation Research Board, vol. 2311, pp. 59-72, 2012.

[2] F. Zheng and H. Van Zuylen, "The development and calibration of a model for urban travel time distributions," Journal of Intelligent Transportation Systems: Technology, Planning, and Operations, vol. 18, no. 1, pp. 81-94, 2014.

[3] W. Pu, "Analytic relationships between travel time reliability measures," Transportation Research Record: Journal of the Transportation Research Board, vol. 2254, pp. 122-130, 2011.

[4] S. Arroyo and A. L. Kornhauser, "Modeling travel time distributions on a road network," in Proceedings of 84th TRB annual meeting, Washington DC, USA, 2005.

[5] H. Al-Deek and E. B. Emam, "New methodology for estimating reliability in transportation networks with degraded link capacities," Journal of Intelligent Transportation Systems: Technology, Planning, and Operations, vol. 10, no. 3, pp. 117-129, 2006.

[6] F. Guo, H. Rakha, and S. Park, "A multi-state travel time reliability model," in Proceedings of the TRB 89th annual meeting, Washington DC, USA, 2010.

[7] P. Chen, K. Yin, and J. Sun, "Application of finite mixture of regression model with varying mixing probabilities to estimation of urban arterial travel times," Transportation Research Record: Journal of Transoportation Research Board, vol. 2442, pp. 96-105, 2014.

[8] S. Clark and D. Watling, "Modelling network travel time reliability under stochastic demand," Transportation Research Part B: Methodological, vol. 39, no. 2, pp. 119-140, 2005.

[9] J. Kim and H. S. Mahmassani, "Compound Gamma representation for modeling travel time variability in a traffic network," Transportation Research Part B: Methodological, vol. 80, pp. 4063, 2015.

[10] J. Kim, H. Mahmassani, P. Vovsha, Y. Stogios, and J. Dong, "Scenario-based approach to analysis of travel time reliability with traffic simulation models," Transportation Research Record: Journal of the Transportation Research Board, vol. 2391, pp. 5668, 2013.

[11] A. Chen and Z. Zhou, "The $\alpha$-reliable mean-excess traffic equilibrium model with stochastic travel times," Transportation Research Part B: Methodologies, vol. 44, no. 4, pp. 493-513, 2010.

[12] J. Bates, J. Polak, P. Jones, and A. Cook, "The valuation of reliability for personal travel," Transportation Research Part E: Logistics and Transportation Review, vol. 37, no. 2-3, pp. 191-229, 2001.

[13] T. Lomax, D. Schrank, S. Turner, and R. Margiotta, Selecting Travel Time Reliability Measures, Texas Transportation Institute, Texas, USA, 2003.
[14] FHWA, Travel Time Reliability: Making It There On Time, All The Time, Federal Highway Administration.

[15] J. W. C. van Lint, H. J. van Zuylen, and H. Tu, “Travel time unreliability on freeways: why measures based on variance tell only half the story," Transportation Research Part A: Policy and Practice, vol. 42, no. 1, pp. 258-277, 2008.

[16] H. K. Lo, X. W. Luo, and B. W. Y. Siu, "Degradable transport network: travel time budget of travelers with heterogeneous risk aversion," Transportation Research Part B: Methodological, vol. 40, no. 9, pp. 792-806, 2006.

[17] X. Xu, A. Chen, L. Cheng, and H. K. Lo, "Modeling distribution tail in network performance assessment: a mean-excess total travel time risk measure and analytical estimation method," Transportation Research Part B: Methodological, vol. 66, pp. 3249, 2014.

[18] E. A. I. Bogers, Traffic Information and Learning in Day-to-Day Route Choice [Dissertation, thesis], Transport \& Planning, Delft University of Technology, 2009.

[19] H. Mahmassani, T. Hou, and J. Dong, "Characterizing travel time variability in vehicular traffic networks: deriving a robust relation for reliability analysis," Transportation Research Record: Journal of Transportation Research Board, no. 2315, pp. 141-152, 2012.

[20] H. Li, Reliability-based Dynamic network Design with Stochastic Networks. Doctoral dissertation [Doctoral, thesis], Delft University of Technology, 2009.

[21] M. Fosgerau and A. Karlström, "The value of reliability," Transportation Research Part B: Methodological, vol. 44, no. 1, pp. 38-49, 2010.

[22] S. Susilawati, M. A. P. Taylor, and S. V. C. Somenahalli, "Distributions of travel time variability on urban roads," Journal of Advanced Transportation, vol. 47, no. 8, pp. 720-736, 2013.

[23] N. L. Johnson, "Systems of frequency curves generated by methods of translation," Biometrika, vol. 36, pp. 149-176, 1949.

[24] J. F. Slifker and S. S. Shapiro, “The johnson system: Selection and parameter estimation," Technometrics, vol. 22, no. 2, pp. 239246, 1980.

[25] N. R. Farnum, "Using Johnson curves to describe non-normal process data," Quality Engineering, vol. 9, no. 2, pp. 329-336, 1996.

[26] E. G. Jones, H. S. Mahmassani, R. Herman, and C. M. Walton, "Travel time variability in a commuting corridor: implications for electronic route guidance," in Proceedings of the the First International Conference on Applications of Advanced Technologies in Transportation Engineering, 1989.

[27] I. D. Hill, R. Hill, and R. L. Holder, "Algorithm AS 99: fitting Johnson curves by moments," Applied Statistics, vol. 25, no. 2, p. 180, 1976.

[28] I. D. Hill, "Algorithm AS 100: Normal-Johnson and Johnson_ Normal transformations," Applied Statistics, vol. 25, no. 2, p. 190, 1976.

[29] D. T. Mage, "An explicit solution for sbparameters using four percentile points," Technometrics, vol. 22, no. 2, pp. 247-251, 1980.

[30] H. Akaike, "A New Look at the Statistical Model Identification," IEEE Transactions on Automatic Control, vol. 19, no. 6, pp. 716723, 1974.

[31] N. Geroliminis and C. F. Daganzo, "Existence of urban-scale macroscopic fundamental diagrams: some experimental findings," Transportation Research Part B: Methodological, vol. 42, no. 9, pp. 759-770, 2008. 
[32] C. F. Daganzo and N. Geroliminis, "An analytical approximation for the macroscopic fundamental diagram of urban traffic," Transportation Research Part B: Methodological, vol. 42, no. 9, pp. 771-781, 2008.

[33] Y. Ji, N. Geroliminis, and J. Luo, "Empirical observations of congestion propagation and dynamic partition with probe data for large scale systems," in Proceedings of the 93rd TRB annual meeting, Washington DC, USA.

[34] L. C. Edie, "Discussion of traffic stream measurements and definitions," in Proceeding of the 2nd International Symposium on the Theory of Traffic Flow, J. Almond, Ed., pp. 139-154, Organisation for Economic Co-operation and Development, 1963.

[35] M. Saberi, H. S. Mahmassani, T. Hou, and A. Zockaie, "Estimating network fundamental diagram using three-dimensional vehicle trajectories: extending edie's definitions of traffic flow variables to networks," Transportation Research Record: Journal of the Transportation Research Board, vol. 2422, pp. 12-20, 2014.

[36] T. Courbon and L. Leclercq, "Cross-comparison of macroscopic fundamental diagram estimation methods," in Proceedings of the Procedia Social and Behavioral Sciences, vol. 20, pp. 417-426, September 2011.

[37] H. Mahmassani, T. Hou, and M. Saberi, "Connecting networkwide travel time reliability and the network fundamental diagram of traffic flow," Transportation Research Record: Journal of the Transportation Research Board, no. 2391, pp. 80-91, 2013. 


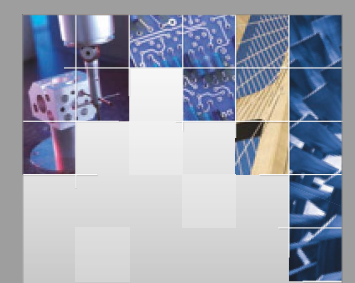

\section{Enfincering}
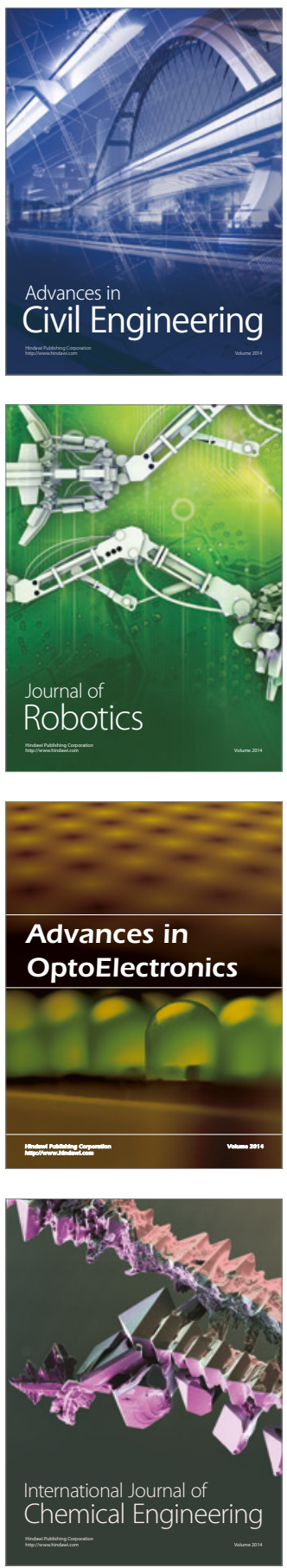

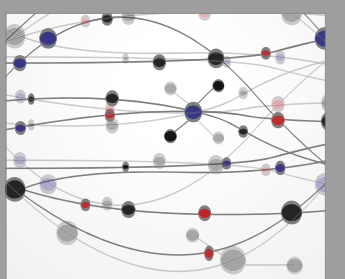

The Scientific World Journal

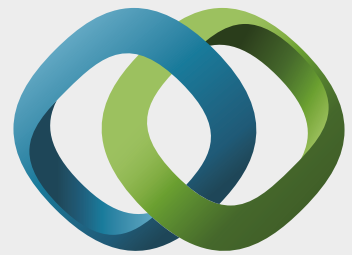

\section{Hindawi}

Submit your manuscripts at

https://www.hindawi.com
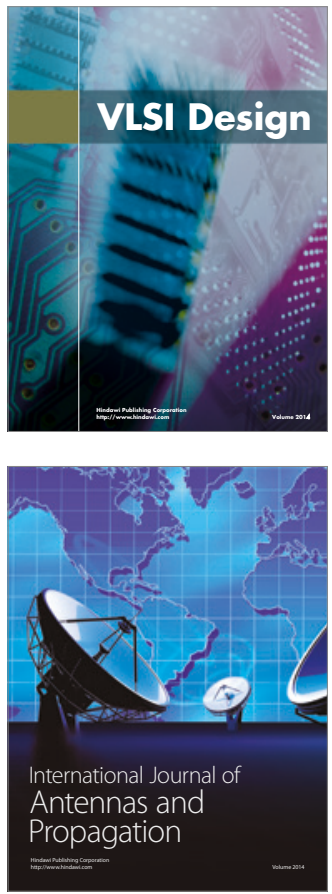

\section{Rotating}

Machinery
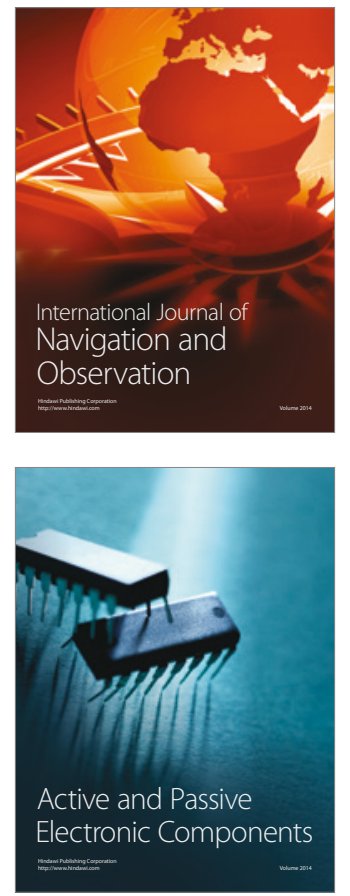
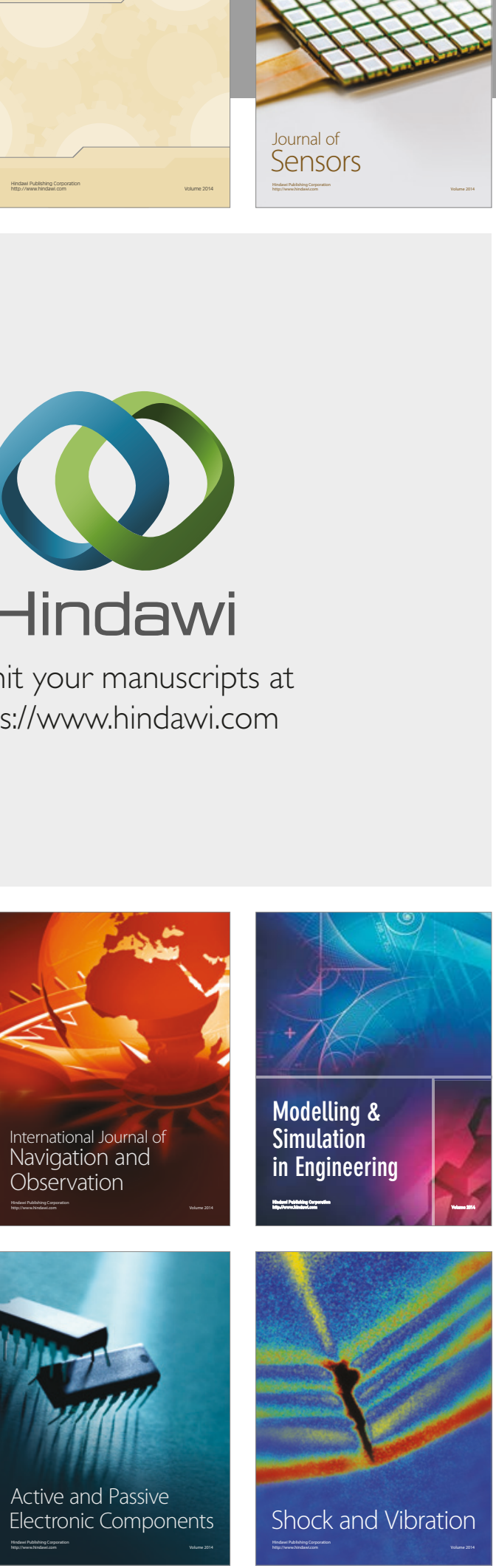
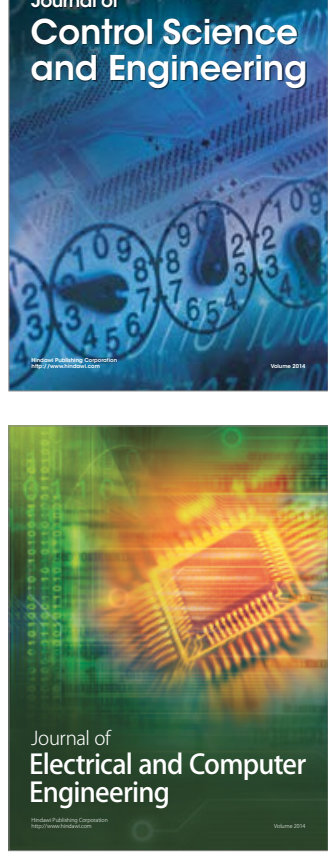

Distributed

Journal of

Control Science

and Engineering
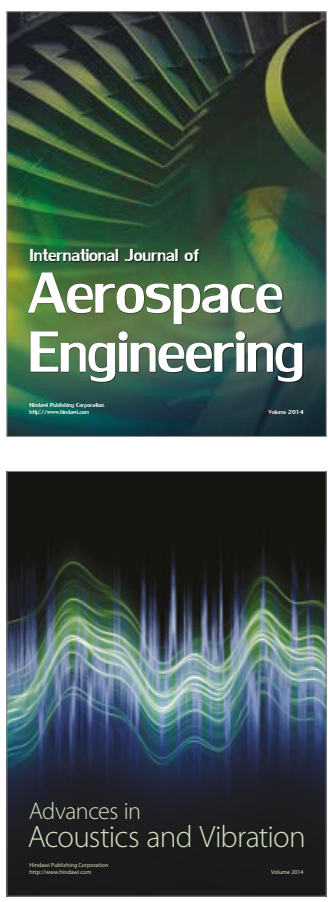

Sensor Networks 